\title{
Design of insulating devices for in vitro synthetic circuits
}

\author{
Elisa Franco, Domitilla Del Vecchio and Richard M. Murray
}

\begin{abstract}
This paper describes a synthetic in vitro genetic circuit programmed to work as an insulating device. This circuit is composed of nucleic acids, which can be designed to interact according to user defined rules, and of few proteins that perform catalytic functions. A model of the circuit is derived from first principle biochemical laws. This model is shown to exhibit time-scale separation that makes its output insensitive to downstream time varying loads. Simulation results show the circuit effectiveness and represent the starting point for future experimental testing of the device.
\end{abstract}

\section{INTRODUCTION}

The functionalities of every living organism are wired in the biochemical interactions existing among proteins, nucleic acids and all the other molecules that constitute life's building blocks. Understanding how to embed any function in this 'hardware of life' via 'molecular programming' is an exciting and challenging task for modern bioengineers and synthetic biologists.

Programming molecules is indeed possible with very high precision when reactions are run in vitro, in a controlled environment with few components. The structural properties of nucleic acids make them ideal programmable molecules to perform molecular algorithms and create logic gates [8], but also to operate as nanodevices with sensory or actuation purposes [10].

Synthetic in vitro genetic transcriptional circuits [4] are built with nucleic acids and few protein species. Despite their simplicity, they can be used as a tool kit to design systems embedding important biological functionalities, such as self repressing or self activating modules, toggle switches and oscillators. Being an interesting benchmark to test new biochemical circuitry designs, it is fundamental to understand how to interconnect different transcriptional modules in a large network, preserving their functionality. Insulation blocks are crucial to this purpose.

Our analysis is also motivated by ongoing research at Caltech and TU Munich, focusing on the interconnection of a synthetic transcriptional oscillator to downstream nanodevices [9]. Both the amplitude and frequency of the oscillations are in fact affected by the presence of high amounts of coupled molecular loads.

Elisa Franco and Richard M. Murray are with the Division of Engineering and Applied Sciences, California Institute of Technology, Pasadena, CA 91125. elisa, murray@cds.caltech.edu

Domitilla Del Vecchio is with the Department of Electrical Engineering and Computer Science at the University of Michigan, Ann Arbor, MI 48109. ddv@eecs.umich.edu

Research supported in part by the Institute for Collaborative Biotechnologies through grant DAAD19-03-D-0004 from the U.S. Army Research Office.
Referring to Fig. $1(a)$ and $(b)$, the objective is that of placing programmable and modular insulating devices between existing signal sources and sinks to be interconnected.

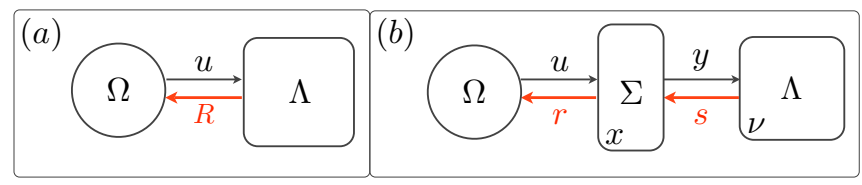

Fig. 1. (a) A system $\Omega$ provides a reference input $u$ to a downstream load system $\Lambda$ : retroactivity effects $R$ could cause distortion of $u$. (b) A properly designed insulation device $\Sigma$ placed between $\Omega$ and $\Lambda$ can reduce the retroactivity signals $r$ and $s$ and guarantee that the dynamics of its output $y$ only depend on $u$.

The concept of retroactivity has been recently proposed in the context of biochemical systems [7], [1], even though interconnecting several devices with minimal node functionality alterations has always been a challenge in many engineering fields. Quantifying analytically the unwanted signals produced within interconnected biological devices is essential to understand how to design and build insulating modules. Time-scale separation is a fundamental tool to this purpose: for instance, protein phosphorylation/dephosphorilation cycles have been proved to minimize their input and output retroactivity [2], thanks to their fast dynamics compared to protein production and degradation.

This paper presents the design of a synthetic transcriptional device that can be used as an insulator to interconnect an input source to a desired load system in the context of in vitro genetic circuits. Such a device is characterized by an input domain, given by single stranded DNA (ssDNA) activators and inhibitors (serving the role of nucleic acid transcription factors), a core represented by a DNA template, and an output RNA transcript. Each domain can be suitably designed for interconnection to arbitrary upstream and downstream modules. Reactions and binding rates of the proposed insulator are programmable through their nucleic acid sequences, and can be tuned to be sufficiently fast to reduce effects of retroactivity to its input and to its output. A model for the device is derived from first principles biochemistry laws and is shown to fulfill the structural properties required to minimize retroactivity effects [2]. A remaining challenge in the design of this synthetic insulator is systematically guaranteeing minimal input/output signal distortion.

In Section II-A we will summarize the technical results from [2] that define the structural properties of biochemical insulators. Section II-B is dedicated to the derivation of a model for the synthetic transcriptional insulator, whose 
input/output decoupling properties are then proved in Section II-C. Finally, numerical simulations showing the behavior of our device interconnected to time varying input and a downstream load are reported in Section III.

\section{DESIGN OF INSULATION DEVICES}

\section{A. Retroactivity: theory background}

Interconnecting several devices without altering their functionality is a challenge in most engineering fields. Classical examples include voltage drops at the output of non-ideal voltage generators, pressure losses in pipe networks or level changes in systems of tanks, which are all effects of the interconnection to a downstream load. In all these cases, the performance degradation is due to unwanted retroactivity phenomena: signals are not transmitted only in the desired direction 'source to load', but can travel back from the load to the source (Fig. 1(a)). Biochemical networks are largely affected by this type of phenomena, since concentrations of chemicals are drained by the reactions in which they participate.

When a dynamical system is interconnected to other upstream and downstream elements, it is generally possible to model the effects of such interconnection through additional system outputs and inputs as shown in Fig. $1(b)$. In general, a system $\Sigma$ having an internal state $x$ can be defined as:

$$
\dot{x}=f(x, u, s), y=Y(x, u, s), r=R(x, u, s),
$$

where $r$ is an additional output representing the effect of $\Sigma$ on upstream systems, while $s$ is an additional input quantifying the effect of downstream systems on $\Sigma$.

An insulating device is a system $\Sigma$ where $r \ll 1, s$ is almost completely attenuated and where the mapping of $u$ to $y$ is linear in some range of interest. Referring to Fig. 1, our objective is that of interconnecting a signal source $\Omega$ and a load system $\Lambda$, through an insulation device $\Sigma$. This device should be designed to provide $\Lambda$ with an input $y$ whose dynamics only depend on the output $u$ of $\Omega$, and to reduce the retroactivity signals $s$ and $r$ that travel back from the load to the signal source. The following structural assumptions [2] are sufficient to demonstrate that the device $\Sigma$ acts as an insulator when interconnected to the source system $\Omega$ and the load $\Lambda$ :

(i) The internal state of $\Sigma$ is $x=\left(x_{1}, \ldots, x_{n}\right) \in \mathcal{D} \subseteq \mathbb{R}_{+}^{n}$, the input $u$ and the output $y=x_{n}$ are both positive scalars, i.e. $u, y \in \mathbb{R}_{+}$. The dynamics of $u$ prior to interconnection are given by

$$
\dot{u}=f_{0}(t, u) \text {. }
$$

The dynamics of $\Sigma$ can be written as

$$
\dot{x}=\left(\begin{array}{c}
G f_{1}(x, u) \\
G f_{2}(x) \\
\vdots \\
G f_{n-1}(x) \\
G f_{n}(x)
\end{array}\right),
$$

and the internal state variables of $\Lambda$ are

$$
\dot{\nu}=\left(\begin{array}{c}
g_{1}(\nu, y) \\
g_{2}(\nu) \\
\vdots \\
g_{p}(\nu)
\end{array}\right) ;
$$

(ii) The variables $r, s$ are scalars and 'parasitically' affect the dynamics of $u$ and $y$ only as additive rates, that is,

$$
\begin{gathered}
\dot{u}=f_{0}(t, u)+r(x, u), \\
\dot{x}_{n}=\dot{y}=G f_{n}(x)+s(\nu, y) ;
\end{gathered}
$$

(iii) The following conservation laws hold for the retroactivity rates:

$$
r(x, u)=-G f_{1}(x, u) \quad \text { and } \quad s(\nu, y)=-g_{1}(\nu, y) .
$$

The parameter $G$ quantifies the speed of the dynamics of $\Sigma$ and plays a fundamental role in reducing the retroactivity to the output $s$. We also need a further assumption regarding the stability properties of $\Sigma$.

Assumption 1: Define $F: \mathbb{R}_{+} \times \mathcal{D} \rightarrow \mathbb{R}^{n}$ as $F(a, x)=$ $\left(f_{1}\left(x, a-x_{1}\right), f_{2}(x), \ldots, f_{n}(x)\right), a \in \mathbb{R}_{+}$, and $x \in \mathcal{D}$. We assume that all the eigenvalues of the Jacobian $D F_{x}(a, x)$ have negative real parts for all $x \in \mathcal{D}$ and all $a \in \mathcal{D}^{\prime}:=$ $\left\{a \in \mathbb{R}_{+} \mid\left(a-x_{1}\right) \geq 0, x \in \mathcal{D}\right\}$.

If the structural assumptions $(i),(i i)$ and (iii) together with Assumption 1 hold, the following claims can be proved [2]:

Claim 1: Let $x(t)$ be generated by the interconnected system (2), (3) and (4), and let $x^{\mathrm{ref}}(t)$ be generated by the same system where we set $s(\nu, y)=0$. Then, there exist constants $G^{*}, t_{0}, T>0$ such that $\left\|x^{\text {ref }}(t)-x(t)\right\|=\mathcal{O}(1 / G)$ for all $t \in\left[t_{0}, T\right)$ and all $G>G^{*}$.

The proof of Claim 1 (we address the reader to [2] for the proof) is based on the application of the singular perturbation theorem on the finite time interval [5]. The global stability of the Jacobian $D F_{x}$ guarantees that the slow manifold is attractive.

Claim 2: Let $u(t)$ be generated by the interconnected system (1)-(5). Define $f(a, x):=\left(f_{1}(x, a), f_{2}(x), \ldots, f_{n}(x)\right)$ and assume that $f(a, \gamma(a))=0$ admits a unique solution $\gamma: \mathbb{R}_{+} \rightarrow \mathbb{R}_{+}^{n}$ with $\gamma(a)=\left(\gamma_{1}(a), \ldots, \gamma_{n}(a)\right)$ and $\gamma_{1}: \mathbb{R}_{+} \rightarrow \mathbb{R}_{+}$. Let $\bar{u}(t)$ be generated by $d \bar{u} / d t=$ $f_{0}(t, u)\left(1 /\left(1+\partial \gamma_{1}(\bar{u}) / \partial \bar{u}\right)\right)$. Then, there exist $G^{*}, t_{0}, T>$ 0 such that $u(t)=\bar{u}(t)+\mathcal{O}(1 / G)$ for all $t \in\left[t_{0}, T\right)$ and all $G>G^{*}$.

Claim 2 (see [2] for the proof) implies that if $\partial \gamma_{1}(\bar{u} / \partial \bar{u}) \ll 1$ then the dynamics of $\bar{u}$ become approximately equal to the dynamics of $\dot{u}=f_{0}(t, u)$ where $r(x, u)=0$. The quantity $\left.\partial \gamma_{1}(\bar{u}) / \partial \bar{u}\right)$ measures the retroactivity to the input $r$ as a function of the input and of the parameters of the insulator $\Sigma$, which can be therefore designed to minimize $r$.

\section{B. Design of an insulation device for in vitro genetic circuits}

We will now describe an insulation device to be used in the context of in vitro synthetic transcriptional circuits. Our device is defined by a set of biochemical species and 
their programmed interactions, which are represented in Fig. 2. In the following we will interchange the notation used to indicate a chemical species and its concentration. For example, let $D_{X}$ denote a certain DNA species; the same symbol will be used to indicate its concentration.

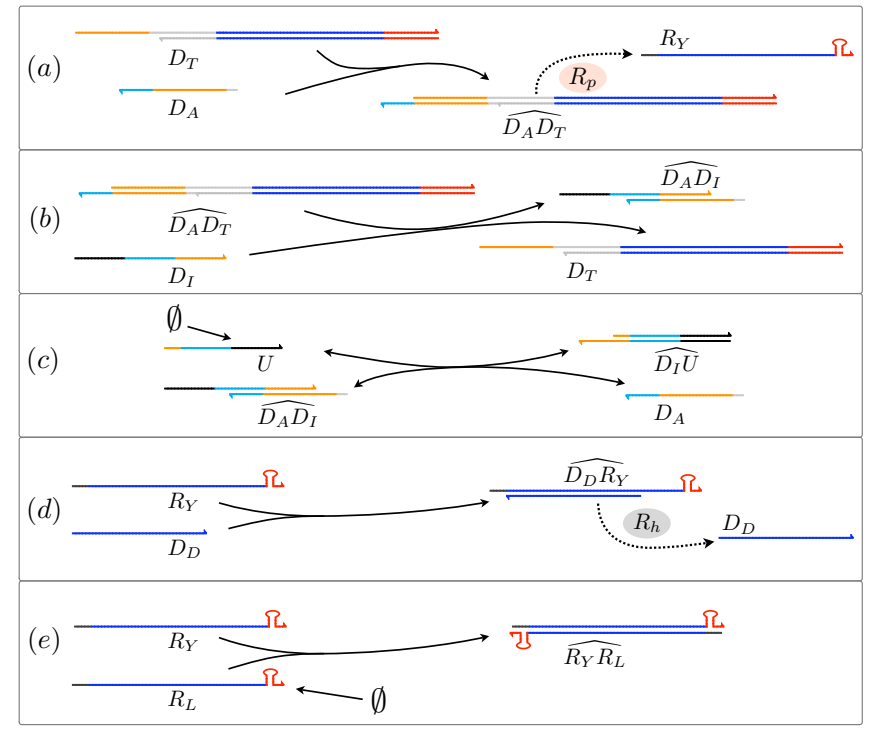

Fig. 2. Reactions occurring in the insulating device. Complementary nucleic acid domains have the same color coding. Different toehold regions are in orange, cyan or black. Blue domains on the templates are transcribed into RNA, and are provided with transcription termination regions, in red. The 5' to 3' orientation is indicated by an arrow on the 3' end of the strands. (a) Activation and transcription. (b) Inhibition. (c) Release of the activator through the input $U .(d)$ Degradation of the RNA output. (e) Output binding to the downstream load $R_{L}$.

The molecular components of the insulating device are five nucleic acids and two enzymes; the process at the core of the device is the control of RNA transcription in a synthetic DNA template $D_{T}$. The promoter region of such template is partially single stranded: efficient transcription is possible only when $D_{T}$ is bound to a DNA activator $D_{A}$ (panel (a) in Fig. 2). The DNA activator can be stripped off the template by a DNA inhibitor $D_{I}$, through the mechanism of branch migration [11] (panel $(b)$ in Fig. 2). The reactions corresponding to this activation/inhibition stage are

$$
\begin{gathered}
D_{A}+D_{T} \stackrel{k_{A T}}{\rightarrow} \widehat{D_{A} D_{T}}, \\
\widehat{D_{A} D_{T}}+D_{I} \stackrel{k_{A I T}}{\rightarrow} D_{T}+\widehat{D_{A} D_{I}}, \\
D_{A}+D_{I} \stackrel{k_{A I}}{\rightarrow} \widehat{D_{A} D_{I}} .
\end{gathered}
$$

The output of the device is the RNA transcript $R_{Y}$, which is produced by the active template complex $\widehat{D_{A} D_{T}}$ in the presence of the enzyme RNA polymerase, $\mathbf{R}_{\mathbf{p}}$ (panel $(a)$ in Fig. 2). Weak binding and transcription occur also when $D_{T}$ is not bound to the activator, but we will assume this contribution is negligible. The production of $R_{Y}$ is thus described by the reactions

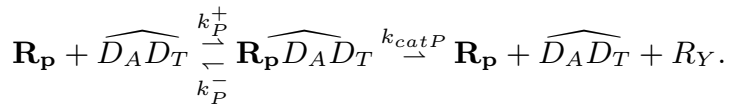

If we assume that the formation of the complex $\mathbf{R}_{\mathbf{p}} \widehat{D_{A} D_{T}}$ is faster than the catalytic step, we can derive the following expression for the production rate of $R_{Y}$ :
$p_{R_{Y}}(t)=k_{c a t P}\left[\mathbf{R}_{\mathbf{p}} \widehat{D_{A} D_{T}}\right]=\frac{k_{c a t P}}{K_{M P}}\left[\mathbf{R}_{\mathbf{p}}{ }^{t o t}\right] \frac{\left[\widehat{D_{A} D_{T}}\right]}{K_{M P}+\left[\widehat{D_{A} D_{T}}\right]}$.

This corresponds to a Hill function of order 1 . We will denote this term as $p_{R_{Y}}(t)=\alpha h\left(\frac{\left[\widehat{D_{A} D_{T}}\right]}{K_{M P}}\right)$. The degradation of the output $R_{Y}$ is mediated by a DNA strand $D_{D}$; the enzyme RNase $\mathrm{H}, \mathbf{R}_{\mathbf{h}}$, will degrade the RNA strand in hybrid DNA-RNA bimolecular complexes (panel $(d)$ in Fig. 2). This represents a tunable negative feedback for the dynamics of $R_{Y}$ :

$$
\begin{aligned}
& D_{D}+R_{Y} \stackrel{k_{D Y}}{\rightarrow} \widehat{D_{D} R_{Y}}, \\
& \mathbf{R}_{\mathbf{h}}+\widehat{D_{D} R_{Y}} \underset{k_{H}^{-}}{\stackrel{k_{H}^{+}}{\rightleftarrows}} \mathbf{R}_{\mathbf{h}} \widehat{D_{D} R_{Y}} \stackrel{k_{\text {cat }}^{\rightarrow}}{\rightarrow} \mathbf{R}_{\mathbf{h}}+D_{D} .
\end{aligned}
$$

The degradation speed of the complex $\widehat{D_{D} R_{Y}}$ is

$$
d_{D_{D} R_{Y}}(t)=\frac{k_{c a t H}}{K_{M H}}\left[\mathbf{R}_{\mathbf{h}}{ }^{t o t}\right] \frac{\left[\widehat{D_{D} R_{Y}}\right]}{K_{M H}+\left[\widehat{D_{D} R_{Y}}\right]} .
$$

This is another Hill function of order 1: $d_{D_{D} R_{Y}}(t)=$ $\gamma h\left(\frac{\left[\widehat{D_{D} R_{Y}}\right]}{K_{M H}}\right)$.

The core of the device is thus represented by the DNA species $D_{T}, D_{A}, D_{I}, D_{D}$ and the RNA output $R_{Y}$.

The synthetic insulator can be interconnected to an input module by designing suitably the inhibitor and activator domains. We will model the input signal $U$ as a DNA species that is introduced in solution at some rate $p_{U}(t)$ and sequestered at some rate $d_{U}(t)$. In particular, the system can be programmed so that its input strand $U$ acts as a releaser molecule for $D_{A}$ making it available again for the activation of $D_{T}$ (panel (c) in Fig. 2). For instance, $U$ could be the output of an arbitrary transcriptional device, which binds and unbinds to the inhibitor species $D_{I}$ and to the complex $\widehat{D_{A} D_{I}}$ releasing $D_{A}$ :

$$
\begin{aligned}
& \emptyset^{p_{U}(t)} U(t) \stackrel{d_{U}(t)}{\rightarrow} \emptyset, \\
& D_{I}+U \underset{k_{I U}^{-}}{\stackrel{k_{I U}^{+}}{D_{I} U},} \\
& \widehat{D_{A} D_{I}}+U^{k_{A I U}} \widehat{D_{I} U}+D_{A} .
\end{aligned}
$$

If the input $U$ is an RNA specie, then the complex $\widehat{D_{I} U}$ would be a substrate for RNase $\mathrm{H}$ : this case is currently being studied, as it introduces undesired coupling between the negative feedback mechanism and the input dynamics of the device. A way to overcome this problem is to work in a saturating regime for the enzyme, but a precise analysis is left as future work.

The device can be interconnected to a downstream module by designing its output to target a molecular load of interest, $R_{L}$. For instance, the load could be represented by another RNA species produced at a certain rate $p_{L}(t)$ (panel $(e)$, Fig. 2):

$$
\begin{aligned}
& \emptyset^{p_{L}(t)} R_{L}(t), \\
& R_{Y}+R_{L} \stackrel{k_{Y L}}{\rightarrow} \widehat{R_{Y} R_{L}} .
\end{aligned}
$$

Given the above set of reactions, it is straightforward to obtain a dynamic model for the DNA insulation device while interconnected to the upstream and downstream systems. 
The ODE model that can be derived by the reactions occurring in the system is:

$$
\begin{aligned}
& \frac{d U}{d t}=+p_{U}(t)-d_{U}(t)-k_{I U}^{+} D_{I} U+k_{I U}^{-} \widehat{D_{I} U} \\
&-k_{A I U} \widehat{D_{A} D_{I}} U \\
& \frac{d \widehat{D_{I} U}}{d t}=+k_{I U}^{+} D_{I} U-k_{I U}^{-} \widehat{D_{I} U}+k_{A I U} \widehat{D_{A} D_{I}} U \\
& \frac{d \widehat{D_{A} D_{I}}}{d t}=+k_{A I} D_{A} D_{I}-k_{A I U} \widehat{D_{A} D_{I}} U \\
& \frac{d \widehat{D_{A} D_{T}}}{d t}=+k_{A T} D_{A} D_{T}-k_{A I T} D_{I} \widehat{D_{A} D_{T}} \\
& \frac{d D_{D}}{d t}=-k_{D Y} D_{D} R_{Y}+\gamma h\left(\frac{\widehat{D_{D} R_{Y}}}{K_{M H}}\right) \\
& \frac{d R_{Y}}{d t}=+\alpha h\left(\frac{\widehat{D_{A} D_{T}}}{K_{M P}}\right)-k_{D Y} D_{D} R_{Y}-k_{Y L} R_{Y} R_{L} \\
& \frac{d R_{L}}{d t} \quad=+p_{L}(t)-k_{Y L} R_{Y} R_{L} .
\end{aligned}
$$

Mass conservation allows us to algebraically derive the concentrations of $D_{A}, D_{T}, D_{I}$ and $D_{D}$ :

$$
\begin{aligned}
& D_{T}^{\mathrm{tot}}=D_{T}+\widehat{D_{A} D_{T}}, \\
& D_{A}^{\text {tot }}=D_{A}+\widehat{D_{A} D_{T}}+\widehat{D_{A} D_{I}}, \\
& D_{I}^{\text {tot }}=D_{I}+\widehat{D_{A} D_{I}}+\widehat{D_{I} U}, \\
& D_{D}^{\text {tot }}=D_{D}+\widehat{D_{D} R_{Y}} .
\end{aligned}
$$

\section{Verification of the insulation properties}

We will now verify that all the structural assumptions defining an insulating device are satisfied by the proposed synthetic module.

First of all, we will assuming that the binding rates of the different DNA species fall in the same order of magnitude. Indeed, the binding rates of nucleic acids can be programmed by specifying their sequence and length: each base-pair match provides known thermodynamic free energy gains and it is possible to predict the binding energetics of arbitrary sequences using existing software packages. Therefore, all the reactions can be designed to have roughly the same speed. Moreover, the amount of enzyme species in solution can be varied to bring the coefficients $\alpha$ and $\gamma$ in the same order of magnitude as the nucleic acid binding rates. Define $G=$ $\max \left\{k_{A T}, k_{A I}, k_{A I T}, k_{I U}^{+}, k_{I U}^{-}, k_{A I U}, k_{D Y}, k_{Y L}, \alpha, \gamma\right\}$, and suppose without loss of generality that $G=k_{A T}$. Then one can define $g_{a t}=k_{A T} / G=1, g_{a i}=k_{A I} / G, g_{a i t}=$ $k_{A I T} / G, g_{i u}^{+}=k_{I U}^{+} / G, g_{i u}^{-}=k_{I U}^{-} / G, g_{a i u}=k_{A I U} / G$, $g_{d y}=k_{D Y} / G, g_{y l}=k_{Y L} / G, \bar{\alpha}=\alpha / G$ and finally $\bar{\gamma}=\gamma / G$, where each of these new rates is of order $\mathcal{O}(1)$.

The device dynamics are easily recast in the same form of the insulator equations (2). In particular the state of the device is defined as $x=\left(\widehat{D_{I} U}, \widehat{D_{A} D_{I}}, \widehat{D_{A} D_{T}}, D_{D}, R_{Y}\right)$, where the output $y=R_{Y}$. Define:

$$
\begin{aligned}
f_{1}(x, u) & =g_{i u}^{+} D_{I} U-g_{i u}^{-} \widehat{D_{I} U}+g_{a i u} \widehat{D_{A} D_{I}} U, \\
f_{2}(x, u) & =g_{a i} D_{A} D_{I}-g_{a i u} \widehat{D_{A} D_{I}} U \\
f_{3}(x) & =D_{A} D_{T}-g_{a i t} D_{I} \widehat{D_{A} D_{T}} \\
f_{4}(x) & =-g_{d y} D_{D} R_{Y}+\bar{\gamma} h\left(\frac{\widehat{D_{D} R_{Y}}}{K_{M H}}\right), \\
f_{5}(x) & =\bar{\alpha} h\left(\frac{\widehat{D_{A} D_{T}}}{K_{M P}}\right)-g_{d y} D_{D} R_{Y} .
\end{aligned}
$$

The dynamics of the input $u(t)=U(t)$ prior to interconnection are given by $\dot{u}=f_{0}(t, u)$, where $f_{0}(t, u)=$ $p_{U}(t)-d_{U}(t)$. The dynamics of the load $\nu=R_{L}$ prior to interconnection are $\dot{\nu}=p_{L}(t)$.

Upon interconnection, the overall system dynamics become:

$$
\begin{aligned}
& \dot{u}=f_{0}(t, u)+r(x, u) \\
& \dot{x}=\left(\begin{array}{c}
G f_{1}(x, u) \\
G f_{2}(x, u) \\
G f_{3}(x) \\
G f_{4}(x) \\
G f_{5}(x)+G s(\nu, y)
\end{array}\right) \\
& \dot{\nu}=f_{\nu}(t)+G s(\nu, y) .
\end{aligned}
$$

We can immediately verify that:

(i) The input $U$ and the output $R_{Y}$ are scalar quantities;

(ii) The variables $r$ and $s$ are scalar additive rates;

(iii) Conservation laws apply to our case: in fact $r(x, u)=$ $-G f_{1}(x, u)$ and $s(\nu, y)=g_{y l} R_{Y} R_{L}$.

Assumption 1 can be verified by computing the Jacobian of $\left.F(a, x)=\left(f_{1}\left(x, a-x_{1}\right), f_{2}\left(x, a-x_{1}\right)\right), \ldots, f_{5}(x)\right)$ for all $x \in \mathcal{D}=\mathbb{R}_{+}^{5}$ and all $a \in \mathcal{D}^{\prime}:=\left\{a \in \mathbb{R}_{+} \mid\left(a-x_{1}\right) \geq\right.$ $0, x \in \mathcal{D}\}$. The Jacobian of $F(x, a)$ has a block structure which is lower diagonal:

$$
D F_{x}(a, x)=\left[\begin{array}{ll}
P & \emptyset \\
L & Q
\end{array}\right] \text {. }
$$

We can find the eigenvalues of $D F_{x}(a, x)$ working on the two blocks $P$ and $Q$ separately. This can be done symbolically as a function of the system parameters and of the equilibria. Let us now assume that $\bar{g}:=g_{i u}^{+} \approx g_{a i u}$, i.e. that the kinetics of the input binding to the complex $\widehat{D_{A} D_{I}}$ are as fast as the kinetics of $U$ binding to unbound $D_{I}$. With this simplification, the elements $p_{i j}$ of matrix $P$ are:

$$
\begin{aligned}
& p_{11}=-G\left(g_{i u}^{-}+\bar{g} D_{I}^{*}+\bar{g} \widehat{D_{I} D_{A}^{*}}+\bar{g}\left(a-\widehat{D_{I} U^{*}}\right)\right) \\
& p_{12}=p_{13}=0 \\
& p_{21}=+G\left(\bar{g} \widehat{D_{I} D_{A}^{*}}-g_{a i} D_{A}^{*}\right), \\
& p_{22}=-G\left(g_{a i}\left(D_{I}^{*}+D_{A}^{*}\right)-\bar{g}\left(a-\widehat{D_{I} U^{*}}\right)\right) \\
& p_{23}=-G g_{a i} D_{I}^{*}, \\
& p_{31}=+G g_{a i t} \widehat{D_{A} D_{T}^{*}}, \\
& p_{32}=-G\left(D_{T}^{*}-g_{a i t} \widehat{D_{A} D_{T}^{*}}\right), \\
& p_{33}=-G\left(g_{a i t} D_{I}^{*}+g_{a i t} D_{T}^{*}+g_{a t} D_{A}^{*}\right) .
\end{aligned}
$$

The index $*$ denotes equilibrium concentrations. Since $p_{12}=$ $p_{13}=0$, the first root of the characteristic polynomial of $P$ is $p_{11}$, which is negative since all the all the binding rates are positive and all equilibrium concentrations are non-negative. The remaining roots are the solution of $\left(\lambda+p_{22}\right)\left(\lambda+p_{33}\right)-$ $\left(p_{23} p_{32}\right)=0$. Since all the coefficients of this polynomial 
are positive at any $x \in \mathcal{D}, a \in \mathcal{D}^{\prime}$, then all the roots of the system have negative real parts.

The coefficients of matrix $Q$ are:

$$
\begin{aligned}
q_{11} & =-G g_{d y} D_{D}^{*}, \\
q_{12} & =-G g_{d y} R_{Y}^{*}-\frac{G \bar{\gamma}}{\widehat{D_{D} R_{Y}^{*}}+K_{M H}}, \\
& +G \bar{\gamma} \frac{\widehat{D_{D} R_{Y}^{*}}}{\left(\widehat{D_{D} R_{Y}^{*}}+K_{M H}\right)^{2}}, \\
q_{21} & =-G g_{d y} R_{Y}^{*} \\
q_{22} & =-G g_{d y} D_{D}^{*} .
\end{aligned}
$$

It is straightforward to verify that also the characteristic polynomial of $Q$ has negative roots at any equilibrium, provided that $K_{M H}>0$.

For a system with the structure (1)-(5), the stability of $D F_{x}(a, x)$ implies the result of Claim 1 . System (11) slightly differs from that structure due to the presence of the timevarying term $f_{\nu}(t)$ in the dynamics of $\nu$. Nevertheless, Claim 1 can be extended to cover this case as follows.

Define $\tilde{u}=u+x_{1}$ and $\varepsilon=1 / G$. In these new variables the dynamics of system (11) become:

$$
\begin{aligned}
\dot{\tilde{u}} & =f_{0}\left(t, \tilde{u}-x_{1}\right)+r\left(x, \tilde{u}-x_{1}\right) \\
\varepsilon \dot{x}_{1} & =f_{1}\left(x, \tilde{u}-x_{1}\right) \\
\varepsilon \dot{x}_{2} & =f_{2}\left(x, \tilde{u}-x_{1}\right) \\
\varepsilon \dot{x}_{3} & =f_{3}(x) \\
\varepsilon \dot{x}_{4} & =f_{4}(x) \\
\varepsilon \dot{x}_{5} & =f_{5}(x)+s\left(\nu, x_{5}\right) \\
\varepsilon \dot{\nu} & =\varepsilon f_{\nu}(t)+s\left(\nu, x_{5}\right) .
\end{aligned}
$$

Let $\varepsilon=0$ in the above system, and let $\gamma(u)=$ $\left(\gamma_{1}(u), \ldots, \gamma_{5}(u)\right)$ be the locally unique solution of $F(\tilde{u}, \gamma(\tilde{u}))=0$. Since all the eigenvalues of $D F_{x}(a, x)$ have negative real parts on all its domain $\mathcal{D}$, the trajectories are attracted by the slow manifold. The singular perturbation theorem over the finite time interval [5] can thus be applied, and there exist $\varepsilon^{\prime}, t^{\prime}$ and $T^{\prime}$, with $t^{\prime}<T^{\prime}$, such that the state $x(t)=\gamma(\tilde{u}(t))+\mathcal{O}(\varepsilon)$ for all $\varepsilon<\epsilon^{\prime}$, and $t \in\left[t^{\prime}, T^{\prime}\right)$. Therefore, the dynamics of $x$ on the slow manifold do not depend on the $\nu$ variable nor on $f_{\nu}(t)$.

Similarly, it is also possible to prove that for $G$ sufficiently large, the solution $x(t)$ will converge to $x^{\mathrm{ref}}(t)$, the state generated by system (13) when $s(\nu, y)=0$. We refer the reader to [2] for a detailed proof.

We now need to verify that Claim 2 holds for our device, namely that we can design our module to present low retroactivity to the input. An analytic solution of the equilibria for system (13) cannot be found in a straightforward manner, though its existence is locally guaranteed by the fact that $F(a, x)$ is a diffeomorphism on $\mathcal{D}^{\prime} \times \mathcal{D}$. An equilibrium for $x_{1}=\widehat{D_{I} U}$ as a function of $U$ can though be found easily if we assume again that $\bar{g}:=g_{i u}^{+} \approx g_{a i u}$. In this case:

$$
0=\bar{g} D_{I} U-g_{i u}^{-} \widehat{D_{I} U}+\bar{g} \widehat{D_{A} D_{I}} U \text {. }
$$

Since $D_{I}=D_{I}^{\text {tot }}-\widehat{D_{A} D_{I}}-\widehat{D_{I} U}$, we can derive

$$
\widehat{D_{I} U^{*}}=D_{I}^{\mathrm{tot}} \frac{U}{\frac{g_{i u}^{-}}{\bar{g}}+U}=\gamma_{1}(U) .
$$

Following the notation of Claim 2, in this case

$$
\frac{\partial \gamma_{1}(U)}{\partial U}=D_{I}^{\text {tot }} \frac{\frac{g_{i u}^{-}}{\bar{g}}}{\left(\frac{g_{i u}^{-}}{\bar{g}}+U\right)^{2}} .
$$

Low retroactivity to the input is achieved if

$$
D_{I}^{\text {tot }} \frac{\frac{g_{i u}^{-}}{\bar{g}}}{\left(\frac{g_{i u}^{-}}{\bar{g}}+U\right)^{2}} \ll 1 .
$$

A simple analytical relationship between the insulator input and output is at present not available. This does not allow us yet to design a device operating in a linear regime, avoiding signal distortion. Further work in this direction is ongoing.

\section{SIMULATION RESULTS}

In this section, the synthetic device described at equations (11) is numerically simulated using the MATLAB ode23t solver. We assume that the device input $U(t)$ is a ssDNA molecule which binds and unbinds to the $D_{I}$ species, releasing the activator $D_{A}$. The device is then connected to a downstream load $R_{L}$. We consider the case in which the load is being produced at some rate $p_{L}$. The utilized kinetic parameters are reported in Table I and fall in the typical range of experimentally measured kinetic rates for nucleic acids and enzymes [4]. The initial conditions for all the cases simulated are in Table II and are also typical of experiments done with in vitro genetic circuits.

The kinetic reaction rates of different nucleic acids are fast, in the order of $10^{4}-10^{6}$ per mole per second. Such rates can be tuned by suitably designing the complementarity regions of the different strands and the lengths of the toehold domains, necessary for the branch migration process. The speed of the insulating device can be therefore specified by sequence design. Numerical algorithms and software packages like Nupack [3] or UNAfold [6] allow us to check the thermodynamic likelihood of formation of the nucleic acid complexes of interest. The kinetics of such reactions can also be estimated using in house software packages (currently under development in the Winfree and Pierce laboratories at Caltech).

The kinetic rates of commercially available enzymes are generally unknown to the end user. The speed of $\mathbf{R}_{\mathbf{p}}$ and $\mathbf{R}_{\mathbf{h}}$ is often the bottleneck in the dynamics achievable with in vitro transcriptional circuits. When designing a new device, it is necessary to search for the optimal concentrations of proteins that can sustain the desired system speed.

We looked at the behavior of the device with minimal enzyme concentrations and with high enzyme concentrations: this is equivalent to modulating the value of $\alpha$ and $\gamma$ in equations (10). The results are shown in Fig. 3. The performance of the device when the enzymatic concentrations are low is unsatisfactory, since the output profile never recovers the reference trajectory, Fig. $3(a)$. If the amounts of enzyme are instead increased ten fold, the trajectory of the output under 
load $R_{Y}$ stays very close to that of $R_{Y}^{\text {ref }}$, Fig. $3(b)$. When increasing the RNase $\mathrm{H}$ concentration, the amount of $D_{D}^{\text {tot }}$ has also been scaled up; empirically this parameter has been observed to cause a shift in the average value of the output oscillation, while it does not have significant effects on its amplitude and frequency.

The retroactivity to the input is minimal, since $D_{I}^{\text {tot }} \ll$ $U^{2}(t)$ for any $t \geq 0$. The distortion from the input $U$ to the output $R_{Y}$, even when $s\left(R_{Y}, R_{L}\right)=0$, is due to the nonlinear nature of the dynamics. Since it is not possible to obtain a simple expression for the equilibria of the device as a function of the input and the initial conditions, it is also impossible to get a systematic insight on what parameters and initial conditions would minimize such input/output distortion.

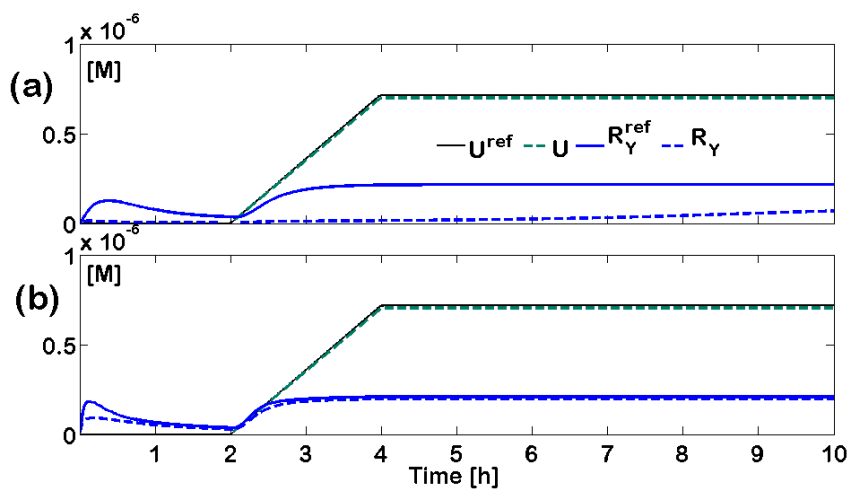

Fig. 3. Performance of the synthetic insulator when the load is slowly increasing over time. (a) Low amount of enzymes: $R_{Y}$ does not recover the load-free reference $R_{Y}$. (b) High amount of enzymes: the error between $R_{Y}$ and $R_{Y}^{r e f}$ is greatly reduced.

TABLE I

SIMULATION PARAMETERS

\begin{tabular}{lll}
\hline Units: $[1 / s M]$ & Units: $[1 / s]$ & Units: $[M]$ \\
\hline$k_{A T}=510^{4}$ & $k_{\text {cat } P}=0.3$ & $k_{M P}=2.50 \cdot 10^{-7}$ \\
\hline$k_{A I}=10^{4}$ & $k_{\text {cat } H}=0.8$ & $k_{M H}=5 \cdot 10^{-8}$ \\
\hline$k_{A I T}=10^{4}$ & $k_{\text {cat } P}=0.2$ & \\
\hline$k_{I U}^{+}=10^{4}$ & Units: $[M / s]$ & Units: $[M / s]$ \\
\hline$k_{I U}^{-}=0.05$ & $\begin{array}{l}p_{U}(t)=a_{0}, 2 h \leq t \leq 4 h \\
p_{U}(t)=0 \text { otherwise }\end{array}$ & $\omega=5.5510^{-4}$ \\
\hline$k_{A I U}=10^{4}$ & $d_{U}(t)=10^{-10}$ & $a_{0}=10^{-10}$ \\
\hline$k_{D Y}=10^{4}$ & $p_{L}=1.2 \cdot 10^{-9}$ & \\
\hline$k_{Y L}=10^{4}$ & & \\
\hline
\end{tabular}

\section{CONCLUSIONS AND FUTURE WORKS}

This paper describes the design of an insulating device to be used in the context of synthetic in vitro transcriptional circuits. Due to their simplicity and high programmability, such circuits are useful to understand and implement general design principles for biochemical networks. Building large scale networks of synthetic circuits relies heavily on the
TABLE II

INITIAL CONDITIONS

\begin{tabular}{|c|c|c|c|c|}
\hline Species & Units: $[n M]$ & & Species & Units: $[M]$ \\
\hline$D_{A}(0)=D_{A}^{\mathrm{tot}}$ & $1.5 \cdot 10^{-7}$ & & $R_{Y}(0)$ & $2 \cdot 10^{-6}$ \\
\hline$D_{I}(0)=D_{I}^{\text {tot }}$ & $1.50 \cdot 10^{-7}$ & & $R_{L}(0)$ & 0 \\
\hline$D_{T}(0)=D_{T}^{\text {tot }}$ & $1.50 \cdot 10^{-7}$ & & $U(0)$ & $10^{-8}$ \\
\hline$D_{D}(0)=D_{D}^{\text {tot }}$ & Low: $2 \cdot 10^{-7}$ & High: $1.6 \cdot 10^{-6}$ & & \\
\hline $\mathbf{R}_{\mathbf{p}}(0)$ & Low: $4 \cdot 10^{-9}$ & High: $40 \cdot 10^{-9}$ & & \\
\hline $\mathbf{R}_{\mathbf{h}}(0)$ & Low: $1 \cdot 10^{-9}$ & High: $10^{-8}$ & & \\
\hline
\end{tabular}

possibility of plugging together several stand alone modules without losing their functionality. Retroactivity effects intrinsically present in biochemical networks can be reduced by employing insulating devices. The modular synthetic insulator described in this paper satisfies the structural requirements to guarantee low retroactivity to its input and output [2]. Currently this device can be interconnected to arbitrary DNA inputs and generic nucleic acid outputs. A design challenge is represented by the fact that enzymatic species are globally shared in transcriptional circuits: working at catalytic saturation (thereby forcing Hill coefficients in their linear regime) is likely the only scalable method do avoid strong overall coupling. Further work is also required to understand how to systematically program the device to present a minimal input/output signal distortion.

\section{ACKNOWLEDGEMENTS}

The authors would like to thank Erik Winfree, Friedrich Simmel and Jongmin Kim for helpful discussions and advise regarding the design and synthesis of in vitro genetic circuits.

\section{REFERENCES}

[1] Del Vecchio, D., Ninfa, A. and Sontag, E. Modular Cell Biology: Retroactivity and Insulation Nature Molecular Systems Biology, 2008, 4, 161

[2] Del Vecchio, D. and Jayanthi, S. Retroactivity Attenuation in Transcriptional Networks: Design and Analysis of an Insulation Device Conference on Decision and Control, Proceedings of the IEEE, Dec. 2008

[3] Dirks, R. M., Bois, J. S., Schaeffer, J. M., Winfree, E. and Pierce, N. A. Thermodynamic analysis of interacting nucleic acid strands SIAM Rev, 2007, 49, 65-88

[4] Kim, J., White, K. S. and Winfree, E. Construction of an in vitro bistable circuit from synthetic transcriptional switches Nature Molecular Systems Biology, 2006, 68

[5] Kokotovic, P., Khalil, H. K. and O'Reilly, J. Singular Perturbation Methods in Control SIAM, 1999

[6] Markham, N. R. and Zuker, M. DINAMelt web server for nucleic acid melting prediction Nucleic Acids Res., 2005, 33, W577-W581.

[7] Saez-Rodriguez, J., Kremling, A., Conzelmann, H., Bettenbrock, K. and Gilles, E. Modular analysis of signal transduction networks Control Systems Magazine, IEEE, Aug. 2004, 24, 35-52

[8] Seelig, G., Soloveichik, D., Zhang, D. Y. and Winfree, E. Enzyme-free nucleic acid logic circuits Science, 2006, 314, 1585-1588

[9] Friedrichs, E., Franco, E., Jungmann, R., Murray, R. M., Kim, J., Winfree, E., and Simmel, F. C. In preparation

[10] Win, M. N. and Smolke, C. D. Higher-Order Cellular Information Processing with Synthetic RNA Devices Science, 2008, 322, 456-460

[11] Yurke, B. and Mills, A. P. Using DNA to Power Nanostructures Genetic Programming and Evolvable Machines, 2003, 4, 111-122 